\title{
Research Paper: Comparison of Pemberton Osteotomy and Kalamchi Modification of Salter Osteotomy in the Treatment of Developmental Dysplasia of the Hip
}

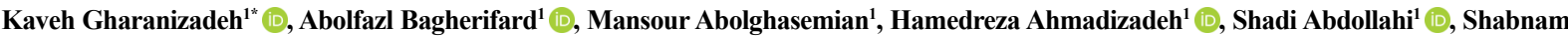
Bayat $^{1}$

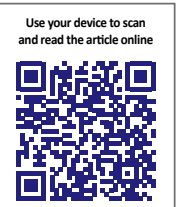

Citztion Gharanizadeh K, Bagherifard A, Abolghasemian M, Ahmadizadeh H, Abdollahi Sh, Bayat Sh. Comparison of Pemberton Osteotomy and Kalamchi Modification of Salter Osteotomy in the Treatment of Developmental Dysplasia of the Hip. Journal of Research in Orthopedic Science. 2020; 7(4):169-174. http://dx.doi.org/10.32598/JROSJ.7.4.727.1

doi: : http://dx.doi.org/10.32598/JROSJ.7.4.727.1

Keywords:

Developmental dysplasia of the hip, Kalamchi modification of Salter osteotomy, Pemberton osteotomy

\begin{abstract}
A B S T R A C T
Background: Several pelvic osteotomy techniques are introduced for the treatment of Developmental Dysplasia of the Hip (DDH). However, there is no agreement on the optimal pelvic osteotomy in DDH. Thus, this study aimed to compare the outcomes of Pemberton Osteotomy (PO) and the Kalamchi Modification of Salter Osteotomy (KMSO).

Objectives: Comparison of pemberton osteotomy and kalamchi modification of salter osteotomy in the treatment of developmental dysplasia of the hip.

Methods: In a retrospective study, radiographic and clinical outcomes as well as surgical complications were compared between the patients who underwent unilateral DDH surgery using either KMSO or PO. The radiographic measures included the assessment of the acetabular index and the Shenton line. The clinical results of the osteotomies were evaluated by McKay's criteria modified by Berkeley et al. Also, Kalamchi and MacEwen's classification was used for the assessment of avascular necrosis.

Results: The characteristic features of the patients, such as age and follow-up time, were statistically comparable between the two study groups. One year after the surgery, the Shenton line was intact in 55 patients (84.6\%) of the KMSO group and 40 patients (88.9\%) of the PO group $(\mathrm{P}=0.52)$. The Mean $\pm \mathrm{SD}$ value of the acetabular index was $21.1 \pm 5.1$ and $20.7 \pm 3.9$ in the KMSO and $\mathrm{PO}$ groups, respectively $(\mathrm{P}=0.13)$. Besides, the McKay's clinical criteria were respectively excellent, good, and fair in 44,16 , and 5 patients $(67.7 \%, 24.6 \%$, and $7.7 \%$, respectively) of the KMSO group, and 31,12 , and 2 patients $(70 \%, 26.6 \%$, and $4.4 \%$, respectively) of the PO group $(\mathrm{P}=0.4)$. Moreover, the number of postoperative avascular necrosis did not significantly differ between the two study groups.
\end{abstract}

Conclusion: The PO and KMSO techniques are equally safe and effective osteotomies in the treatment of DDH and can be used interchangeably.

\footnotetext{
* Corresponding Author:

Kaveh Gharanizadeh, MD.

Address: Bone and Joint Reconstruction Research Center, Shafa Yahyaeian Hospital, Iran University of Medical Sciences, Tehran, Iran.

Phone: +98 (21) 33542000-8

E-mail: kaveh.gharani@gmail.com
} 


\section{Introduction}

evelopmental Dysplasia of the Hip (DDH) is a common congenital disorder $\mathbf{D}$ with an approximate incidence of 12 per 1000 Iranian neonates [1]. It is characterized by the incompatibility of the femoral head and socket, causing several pathologies, such as a hip joint instability or subluxation, the irreducible dislocation of the hip, and the dysplasia of the femoral head and acetabulum $[2,3]$.

Below the age of 18 months, closed reduction and fixation with spica cast is the treatment of choice for DDH. After the age of 18 months, the standard choice of treatment includes open reduction and hip recon-struction using osteotomy techniques [4]. A femoral or pelvic osteotomy or a combination of both is employed, depending on the nature of dysplasia [5].

A pelvic osteotomy is indicated when the femoral head holds insufficient coverage. The Salter Osteotomy (SO) and Pemberton Osteotomy (PO) are the most popular pelvic osteotomy procedures $[5,6]$. The $\mathrm{SO}$ is also known as the Salter Innominate Osteotomy (SIO) that comprises an open-wedge osteotomy with cutting the pelvis and rotating the entire socket around a fixed axis to provide the femoral head with a better coverage [7]. Besides, the PO entails creating a wedge above the acetabulum, thereby, it redirects the acetabular roof and increases the coverage of the femoral head [8].

Despite the favorable results of SO in the management of DDH, it was associated with occasional medial and posterior displacement and limb length discrepancy. To remove these shortcomings, Kalamchi intro-duced a modified version of $\mathrm{SO}$, in which a posterior triangular area was resected from the proximal aspect of the osteotomy site to permit the involvement of the distal iliac section after the displacement of the osteotomized segment [9]. The subsequent investigation reported the successful results of these modifications [10].

Previous investigations have shown the superior results of $\mathrm{PO}$ in comparison with $\mathrm{SO}$ in the treatment of DDH $[11,12]$. To the best of our knowledge, no study has compared the outcomes of the PO and the Kalamchi modification of Salter Osteotomy (KMSO) in DDH patients.

The identification of the optimal pelvic osteotomy technique is necessary to ensure the most effective and the least complicated outcomes in DDH patients. This study compares the outcomes of KMSO and PO in the treatment of DDH to specify the procedure with superior outcomes.

\section{Materials and Methods}

This retrospective cohort study was approved by the Institutional Review Board of our university. We evaluated the eligibility criteria in patients who underwent unilateral DDH surgery using either KMSO or PO, in our university hospital, between 2007 and 2019. The inclusion criteria were a minimum follow-up of one year and complete radiologic records. The exclusion criteria were neurological and syndromic diseases and the history of lower limb surgery, such as combined femoral shortening. Also, patients who were lost to follow-up were excluded from the study.

The demographic characteristics of the patients were extracted from their medical profiles and included age, gender, age at the surgery, and the age of plastering. Furthermore, surgical complications, such as Avascular Necrosis $(\mathrm{AVN})$ were extracted from the patients' profiles. Then, Kalamchi and MacEwen's classification was used for the assessment of AVN. Accordingly, the AVN was classified into four grades: grade I (alterations in the ossific nucleus), grade II (lateral physeal damage), grade III (central physeal damage), and grade IV (total damage) [13].

Radiographic measures were evaluated on the radiographic records of before the surgery, immediately after the surgery, and one year after the surgery and included the Assessment of Acetabular Index (AI) and the Shenton line.

The clinical results of the osteotomies were evaluated by McKay's criteria modified by Berkeley et al. Accordingly, clinical outcomes were categorized into five grades: excellent (painless and stable hip with no limping and $15^{\circ}$ of internal rotation), good (painless and stable hip with slight liming or decreased internal rotation and negative Trendelenburg's sign), fair (moderate pain and stiffness and positive Trendelen-burg's sign), and poor (significant pain) [14]. All the measurements were done by a single observer who was not involved in clinical care.

\section{Surgical procedures and postoperative protocol}

The KMSO and PO were performed as primarily described by Kalamchi [9] and Pemberton [15], respectively. Also, all the surgeries were performed by the same surgeon. Six weeks after the operation, the spica cast was replaced by a broomstick cast in a neutral hip position. At this time, the patients were advised to do hip range-of-motion exercises in all directions as much 
as the cast allowed. Also, standing and weight-bearing exercises were recommended. After one month, the cast was removed, and walking was started. Moreover, three months of physiotherapy and gait training was administered to increase hip range-of-motion.

\section{Statistical analysis}

The obtained data were analyzed with SPSS for Windows (version 16). Initially, the normal distribution of the data was evaluated by the Shapiro-Wilk test. Then, the mean scores were compared between the two osteotomy groups using an independent $t$ test for normally distributed variables or Mann-Whitney U test for non-normally distributed variables. Also, the Chi-square or Fisher's exact test was used to analyze the qualitative data. In all analyses, a $\mathrm{P}$ value of below 0.05 was considered significant.

\section{Results}

A total number of 110 patients with DDH were included in this study. The KMSO and PO were used in 65 patients $(59.1 \%)$ and 45 patients $(40.9 \%)$, respectively. The Mean \pm SD age of the patients was $30.6 \pm 17.4$ months in the KMSO group and $31.3 \pm 18.9$ months in the PO group. However, the age difference was not statistically significant $(\mathrm{P}=0.4)$. The Mean $\pm \mathrm{SD}$ follow-up time of the patients was $54.6 \pm 21.8$ and $52.8 \pm 21.9$ months in the KMSO and PO groups, respectively. Though, the groups did not significantly differ in this regard $(\mathrm{P}=0.18)$. Also, no other significant difference was found between the characteristic features of the two study groups (Table 1).

The Mean \pm SD preoperative AI was $40.7 \pm 7.7^{\circ}$ in the KMSO group and $39.5 \pm 5.9^{\circ}$ in the $\mathrm{PO}$ group $(\mathrm{P}=0.09)$. Immediately after the surgery, the Mean $\pm \mathrm{SD}$ AI was $24.6 \pm 5.3^{\circ}$ and $23.8 \pm 4.9^{\circ}$ in the KMSO and PO groups, respectively $(\mathrm{P}=0.11)$. Also, one year after the surgery, the mean \pm SD AI was $21.1 \pm 5.1^{\circ}$ in the KMSO group and $20.7 \pm 3.9^{\circ}$ in the $\mathrm{PO}$ group $(\mathrm{P}=0.13)$.

Before the surgery, the Shenton line was disrupted in 64 patients $(98.4 \%)$ of the KMSO group and all patients of the PO group $(\mathrm{P}=0.98)$. One year after the surgery, the Shenton line was intact in 55 patients $(84.6 \%)$ of the KMSO group and 40 patients $(88.9 \%)$ of the $\mathrm{PO}$ group $(\mathrm{P}=0.52)$.

Based on the McKay's criteria modified by Berkeley et al., the clinical results of the patients were respectively excellent, good, and fair in 44,16 , and 5 patients $(67.7 \%$, $24.6 \%$, and $7.7 \%$, respectively) of the KMSO group, and 31,12 , and 2 patients $(70 \%, 26.6 \%$, and $4.4 \%$, respectively) of the PO group. This difference was statistically

Table 1. Comparison of the characteristic features of the two study groups

\begin{tabular}{|c|c|c|c|c|}
\hline & & \multicolumn{2}{|c|}{ 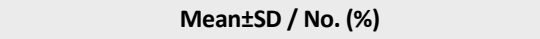 } & \multirow{2}{*}{$\mathrm{P} * *$} \\
\hline \multicolumn{2}{|c|}{ Variable } & KMSO Group $(n=65)^{*}$ & PO Group $(n=45)^{*}$ & \\
\hline \multicolumn{2}{|c|}{ Age (mo) } & $30.6 \pm 17.4$ & $31.3 \pm 18.9$ & 0.4 \\
\hline \multirow{3}{*}{ Gender } & Male & $9(13.8)$ & $3(6.7)$ & \multirow{2}{*}{0.26} \\
\hline & Female & $58(86.2)$ & $42(93.3)$ & \\
\hline & Follow-up (mo) & $54.6 \pm 21.8$ & $52.8 \pm 21.9$ & 0.18 \\
\hline \multirow{2}{*}{ Laterality } & Right & $41(63.1)$ & $28(62.3)$ & \multirow{2}{*}{0.69} \\
\hline & Left & $24(36.9)$ & $17(37.7)$ & \\
\hline \multirow{2}{*}{ Subtrochanteric type } & Shortening & $14(21.5)$ & $13(28.9)$ & \multirow{2}{*}{0.38} \\
\hline & Derotation & $51(78.50$ & $32(71.1)$ & \\
\hline \multirow{2}{*}{ Reduction } & Open & 63 (96.9) & $44(100)$ & \multirow{2}{*}{0.51} \\
\hline & Closed & $2(3.1)$ & $0(0)$ & \\
\hline \multirow{4}{*}{ Dysplasia } & Low & $10(15.6)$ & $10(22.2)$ & \multirow{4}{*}{0.1} \\
\hline & High & $22(34.4)$ & $9(20)$ & \\
\hline & Pseudoacetabulum & $4(6.3)$ & $14(31.8)$ & \\
\hline & Lateralized & $28(43.8)$ & (26) & \\
\hline
\end{tabular}

KMSO: Kalamchi modification of Salter osteotomy; PO: Pemberton osteotomy;

Orthopedic Science

* Data are presented as mean \pm SD or number $(\%)$;

** The $\mathrm{P}$ value of below 0.05 is considered significant. 
significant $(\mathrm{P}=0.4)$. Also, no poor result was seen in the patients of the two study groups.

\section{Postoperative complications}

Immediately after the surgery, two patients of the KMSO group and no patient of the PO group had dislocation $(\mathrm{P}=0.52)$. Moreover, two patients of the KMSO group required a re-reduction during the year after the surgery, while no patient of the PO group required a rereduction during the same period.

In the last follow-up session, the AVN grades I, II, III, and IV were observed respectively in $8,5,2$, and 1 patient(s) $(12.3 \%, 7.7 \%, 3.1 \%$, and $1.5 \%$, respectively) of the KMSO group. Again, the AVN grades I, II, and III were observed seen respectively in 5,4 , and 2 patients $(11.1 \%, 6.2 \%$, and $4.4 \%$, respectively) of the PO group. However, the AVN grade IV was not seen in the PO group. The number of AVN did not significantly differ between the KMSO and $\mathrm{PO}$ groups $(\mathrm{P}=0.33)$.

\section{Discussion}

A wide variety of pelvic osteotomies have been introduced for the treatment of DDH. However, there is no agreement on the optimal pelvic osteotomy for these patients, and comparative studies are required to identify the most efficacious option. Therefore, this study compared the radiologic and clinical outcomes and surgical complications of the DDH patients treated by the KMSO and PO techniques. According to our results, the radiologic outcomes, including the Shenton line and AI did not significantly differ between the two study groups. The clinical results of the patients were comparable between the two study groups. Also, no significant difference was found between the surgical complications of the two osteotomy techniques.

Several studies have evaluated the efficacy of PO versus SO in the treatment of DDH. Ezirmik and Yildiz compared the outcome of SIO and PO in the treatment of 126 hips of $63 \mathrm{DDH}$ patients. The mean cor-rection of AI was $18.3^{\circ}$ and $25.8^{\circ}$ in the SIO and PO groups, respectively. Also, the mean correction of the center-edge angle was $39.15^{\circ}$ in the SIO group and $43.11^{\circ}$ in the PO group. The mean correction of the cervico-diaphyseal angle was $9.22^{\circ}$ in the SIO group and $8.62^{\circ}$ in the PO group. Moreover, the clinical results (based on the Modified McKay Grading System) were excellent in $90.9 \%$ of the patients of the SIO group and $91.8 \%$ of the patients of the PO group. The patients of the SIO group had a $0.47 \mathrm{~cm}$ lengthening of the involved limb. Although the number of AVN was more in the SIO group, the range of motion, the cervico-diaphyseal angle, and the Sharp angle were slightly better in this group. Finally, the authors concluded that the PO provides better femoral head coverage than the $\mathrm{SIO}$, and suggested the $\mathrm{PO}$ as a superior choice of osteotomy for the treatment of DDH [11]. In the present study, the mean AI correction was $18.8^{\circ}$ in the PO group. Also, $70 \%$ of the patients revealed an excellent clinical result. Therefore, the outcome of the PO group of the present series was not as good as that of the Ezirmik and Yildiz. This difference could be attributed to the characteristic of the two study groups.

Bibiana and Gregorio compared the outcomes of PO $(n=47)$ and SO $(n=49)$ in the treatment of DDH. They observed the correction of the dysplasia with normal angle values in 40 hips (83\%) and 22 hips (45\%) of the PO and $\mathrm{SO}$ groups, respectively. However, the postoperative AVN was presented in $23 \%$ of the SO group and $13 \%$ of the PO group. Also, cartilage closure was more frequent in the PO group (52\%). These researchers concluded that PO achieves better and longer acetabular coverage, despite a higher rate of closed triradiate cartilage [16] We did not evaluate the cartilage closure in the present series. The PO group of the present study had an AVN rate of $21.7 \%$, which is more than that of Bibiana and Gregorio's study. In the present study, the majority of the AVNs were classified as grade 1 (according to Kalamchi and MacEwen's classification), while Bibiana and Gregorio did not classify the AVNs.

Ertürk et al. compared the radiologic outcomes of SO $(n=47)$ and PO $(n=50)$ in the treatment of DDH. The PO group of their study showed significantly greater changes in the acetabular depth ratio. Howev-er, the AI, the center-edge angle, and Reimer's index did not significantly differ between the two study groups. They concluded that PO unlike SO would result in an improved radiological outcome [17]. The radiologic measures of the present study included the Shenton line and AI. Therefore, the radiologic outcomes of the present series cannot be compared with the study of Ertürk et al.

The review of earlier investigations suggests $\mathrm{PO}$ as a more efficacious method than SO in the treatment of DDH. Removing the posterior triangular area from the proximal side of the osteotomy site, Kalamchi aimed to provide increased stability, prevent occasional medial and posterior displacement, and eliminate the added limb length seen with the SO. Their preliminary results revealed excellent outcomes in patients with unilateral dysplasia and limb-length discrepancy [9]. 
Later, Synder et al. evaluated the outcomes of KMSO in a larger series of DDH patients ( $n=16$ hips). Based on McKay's criteria for clinical results, excellent or good results were seen in $93 \%$ of hips. Further-more, the excellent radiologic outcome was observed in $97 \%$ of the patients, based on the modification of Severin's classification for radiographic evaluation. The mean preoperative and postoperative $\mathrm{AI}$ were $32^{\circ}$ and $15.9^{\circ}$, respectively. Accordingly, the AI correction was $16.1^{\circ}$. However, one partial loss of position and two AVNs were recorded as the complications [10]. In the KMSO group of the present series, the mean correction of AI was $19.6^{\circ}$, which is greater than that of Synder et al. Though, the clinical results were excellent or good in $92.3 \%$ of the patients of the KMSO group, which is consistent with the results of Synder et al.

Several other investigations have compared the PO with other osteotomy techniques [18-20]. To the best of our knowledge, no previous investigation has compared the outcomes of PO and KMSO in the treat-ment of DDH. However, the present study revealed the comparable results of $\mathrm{PO}$ and $\mathrm{KMSO}$ in the treatment of $\mathrm{DDH}$. This result suggests the implication of both osteotomy methods with no safety and efficacy concerns.

The main limitation of this study was its retrospective design. Therefore, future prospective studies are required to confirm the results of the present study.

\section{Conclusion}

The clinical and radiologic outcomes of the PO and KMSO were comparable in the treatment of DDH. The number of complications, such as AVN did not significantly differ between the two osteotomy groups. These results suggest that $\mathrm{PO}$ and KMSO techniques are equally safe and effective osteotomies in the treatment of DDH.

\section{Ethical Considerations}

\section{Compliance with ethical guidelines}

The results described in this paper were part of student thesis. The project was approved in Ethic Committee of Iran University of Medical Sciences (Ethical Code: IR.IUMS. REC. 1397.032).

\section{Funding}

This research did not receive any specific grant from funding agencies in the public, commercial, or not-forprofit sectors.

\section{Authors' contributions}

Conceptualization, study design: Kaveh Gharanizadeh, Abolfazl Bagherifard, Mansour Abolghasemian; Data acquisition, statistical analysis: Shadi Vaziri Kordkandi, Shabnam Bayat; Writing - original draft: Kaveh Gharanizadeh, Abolfazl Bagherifard, Shadi Vaziri Kordkandi; Writing - review $\&$ editing the final version of the manuscript: All authors.

\section{Conflict of interest}

The authors declared no conflict of interest.

\section{Acknowledgments}

The authors would like to express gratitude to the Vice Chancellor for research of Iran University of Medical Sciences.

\section{References}

[1] Vafaee AR, Baghdadi T, Baghdadi A, Khalili Jamnani R. DDH epidemiology revisited: Do we need new strategies? Arch Bone Jt Surg. 2017; 5(6):440-2. [PMID] [PMCID]

[2] Basit S, Hannan MA, Khoshhal KI. Developmental dysplasia of the hip: Usefulness of next generation genomic tools for characterizing the underlying genes - a mini review. Clin genet. 2016; 90(1):16-20. [DOI:10.1111/cge.12755] [PMID]

[3] Aronsson DD, Goldberg MJ, Kling Jr TF, Roy DR. Developmental dysplasia of the hip. Pediatrics. 1994; 94(2 Pt 1):201-8. [PMID]

[4] Kotlarsky P, Haber R, Bialik V, Eidelman M. Developmental dysplasia of the hip: What has changed in the last 20 years? World J Orthop. 2015; 6(11):886-901. [DOI:10.5312/wjo. v6.i11.886] [PMID] [PMCID]

[5] Gulati V, Eseonu K, Sayani J, Ismail N, Uzoigwe C, Choudhury MZ, et al. Developmental dysplasia of the hip in the newborn: A systematic review. World J Orthop. 2013; 4(2):32-41. [DOI:10.5312/wjo.v4.i2.32] [PMID]

[6] Noordin S, Umer M, Hafeez K, Nawaz H. Developmental dysplasia of the hip. Orthop Rev (Pavia). 2010; 2(2):e19. [DOI:10.4081/or.2010.e19] [PMID] [PMCID]

[7] Pekmezci M, Yazici M. [Salter osteotomy: An overview (Turkish)]. Acta Orthop Traumatol Turc. 2007; 41(Suppl 1):37-46. [PMID]

[8] Faciszewski T, Kiefer GN, Coleman SS. Pemberton osteotomy for residual acetabular dysplasia in children who have congenital dislocation of the hip. J Bone Joint Surg Am. 1993; 75(5):643-9. [DOI:10.2106/00004623-199305000-00002] [PMID]

[9] Kalamchi A. Modified Salter osteotomy. J Bone Joint Surg Am. 1982; 64(2):183-7. [PMID] 
[10] Synder M, Forlin E, Xin S, Bowen JR. Results of the Kalamchi modification of salter osteotomy in the treatment of developmental dysplasia of the hip. J Pediatr Orthop. 1992;12(4):449-53. [DOI:10.1097/01241398-199207000-00006] [PMID]

[11] Ezirmik N, Yildiz K. A Biomechanical Comparison between Salter Innominate Osteotomy and Pemberton Pericapsular Osteotomy. Eurasian J Med. 2012; 44(1):40-2. [DOI:10.5152/ eajm.2012.08] [PMID] [PMCID]

[12] Ertürk C, Altay MA, Işikan UE. A radiological comparison of Salter and Pemberton osteotomies to improve acetabular deformations in developmental dysplasia of the hip. J Pediatr Orthop B. 2013; 22(6):527-32. [DOI:10.1097/ BPB.0b013e32836337cd] [PMID]

[13] Kalamchi A, MacEwen GD. Avascular necrosis following treatment of congenital dislocation of the hip. J Bone Joint Surg Am. 1980; 62(6):876-88. [DOI:10.2106/00004623198062060-00002] [PMID]

[14] Berkeley ME, Dickson JH, Cain TE, Donovan MM. Surgical therapy for congenital dislocation of the hip in patients who are twelve to thirty-six months old. J Bone Joint Surg Am. 1984; 66(3):412-20. [DOI:10.2106/00004623-19846603000014] [PMID]

[15] Pemberton PA. Pericapsular osteotomy of the ilium for treatment of congenital subluxation and dislocation of the hip. J Bone Joint Surg Am. 1965; 47(1):65-86. [DOI:10.2106/00004623-196547010-00004] [PMID]

[16] Bibiana DR, Gregorio CTJ. Comparison results between patients with developmental hip dysplasia treated with either salter or pemberton osteotomy. Ortho Res Online J. 2017; 1(4). [DOI: 10.31031/OPROJ.2017.01.000519]

[17] Ertürk C, Altay MA, Isikan UE. A radiological comparison of Salter and Pemberton osteotomies to improve acetabular deformations in developmental dysplasia of the hip. J Pediatr Orthop B. 2013; 22(6):527-32. [DOI:10.1097/ BPB.0b013e32836337cd] [PMID]

[18] Wang C-W, Wu K-W, Wang T-M, Huang S-C, Kuo KN. Comparison of acetabular anterior coverage after Salter osteotomy and Pemberton acetabuloplasty: A long-term follow-up. Clin Orthop Relat Res. 2014; 472(3):1001-9. [DOI:10.1007/s11999-013-3319-6] [PMID] [PMCID]

[19] Badrinath R, Bomar JD, Wenger DR, Mubarak SJ, Upasani VV. Comparing the Pemberton osteotomy and modified San Diego acetabuloplasty in developmental dysplasia of the hip. J child Orthop. 2019; 13(2): 172-9. [DOI:10.1302/18632548.13.190004] [PMID] [PMCID]

[20] Caffrey JP, Jeffords ME, Farnsworth CL, Bomar JD, Upasani VV. Comparison of 3 pediatric pelvic osteotomies for acetabular dysplasia using patient-specific 3D-printed models. J Pediatr Orthop. 2019; 39(3):e159-64. [DOI:10.1097/ BPO.0000000000001271] [PMID] 